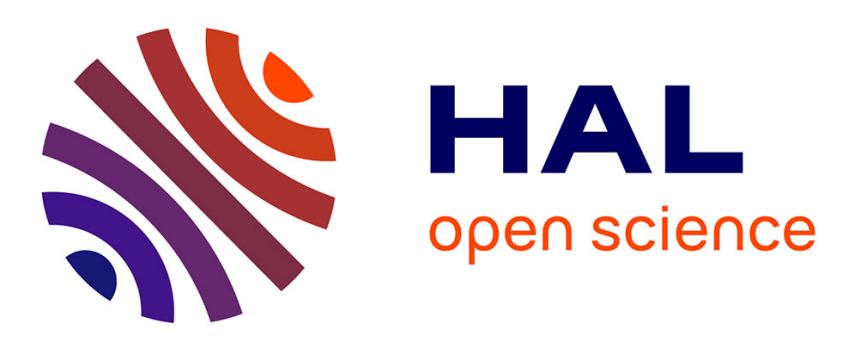

\title{
A Confidence Indicator Model for Virtual Organization Creation in Cloud Computing Environment
}

\author{
Luís Felipe Bilecki, Adriano Fiorese
}

\section{To cite this version:}

Luís Felipe Bilecki, Adriano Fiorese. A Confidence Indicator Model for Virtual Organization Creation in Cloud Computing Environment. 17th Working Conference on Virtual Enterprises (PRO-VE), Oct 2016, Porto, Portugal. pp.200-211, 10.1007/978-3-319-45390-3_18. hal-01614599

\author{
HAL Id: hal-01614599 \\ https://hal.inria.fr/hal-01614599
}

Submitted on 11 Oct 2017

HAL is a multi-disciplinary open access archive for the deposit and dissemination of scientific research documents, whether they are published or not. The documents may come from teaching and research institutions in France or abroad, or from public or private research centers.
L'archive ouverte pluridisciplinaire HAL, est destinée au dépôt et à la diffusion de documents scientifiques de niveau recherche, publiés ou non, émanant des établissements d'enseignement et de recherche français ou étrangers, des laboratoires publics ou privés. 


\title{
A Confidence Indicator Model for Virtual Organization Creation in Cloud Computing Environment
}

\author{
Luís Felipe Bilecki ${ }^{1}$ and Adriano Fiorese ${ }^{1}$ \\ ${ }^{1}$ Department of Computer Science, \\ Santa Catarina State University, Joinville, Brazil \\ luis.bilecki@gmail.com, adriano.fiorese@udesc.br
}

\begin{abstract}
A Virtual Organization (VO) is a form of organization where a set of independent entities share resources, skills and risks in order to attend a collaboration opportunity. The integration between VO's concept and cloud computing seems promising since VO participants use or provide their services in a cloud computing environment. Nevertheless, in this context of integration, the problem of trust between VO partners and cloud providers is highlighted. This is noteworthy since it's necessary to select a reliable cloud provider for a VO business partner launch its service. Therefore, this work presents a confidence indicator model for cloud computing providers using the Data Envelopment Analysis (DEA) method. For the evaluation of this work, we used Quality of Service indicators of public cloud service providers. Results presented and discussed show the generated confidence indicator can be a good criterion to help the VO's manager to select reliable Cloud Computing providers to host VO partners during the VO creation process.
\end{abstract}

Keywords: Virtual Organization, Confidence, Cloud Computing.

\section{Introduction}

In recent years, an increasing variety of collaborative networks have arisen because of the socio-economic challenges faced by society, such as globalization, competitiveness, variety of products and services, low cost, etc [1]. The companies (entities) have been specializing and collaborating with others in order to survive to the competitiveness imposed by other members present in market. In this regard, the collaborative process has became easier due to the advances provided by the information and communication technology (ICT), providing alternatives to deal with the geographical arrangement of the entities [2].

Considering the forms of collaboration among enterprises, Virtual Organization (VO), is advantageous to incorporate flexibility and dynamism to handle the demands of the market [3]. A VO is the temporary alliance of autonomous, heterogeneous and geographically dispersed entities sharing skills and resources to attend a collaboration opportunity (CO). Given that, the VO's life cycle presents the following phases: creation, operation, evolution and dissolution [4]. This article focuses primarily on the creation phase, which is seen as a critical stage to ensure the success of the VO's operation [5]. 
One of the problems observed during the creation of a VO is the necessary sense of confidence among the entities involved, particularly in the sub-process of search and selection [6]. In a VO and Cloud Computing (CC) integration model, cloud computing providers can host VO members' services. In this sense, a VO depends on the cloud providers who host its members' services. Thus, comprising the integration of $\mathrm{VO}$ and Cloud Computing, confidence is a key concept to be taken into consideration during the cloud provider search and selection step in order to form a VO. Moreover, regarding this integration model, the Quality of Service (QoS) delivered by the cloud computing provider plays an important role in the confidence assessment. Therefore, confidence also can help to avoid the dissolution of the VO during the operation phase. Namely, it seems plausible that the greater the confidence level in a cloud computing provider the lower the risk level during the attendance of the collaboration opportunity. This claim is partially supported by results from multicriteria risk assessment works that take into consideration, among other indicators, the confidence one [3, 7].

Therefore, this paper proposes a model for the generation of the confidence indicator, through the application of the Data Envelopment Analysis (DEA) decisionmaking method. Considering that there are multiple inputs represented by Quality of Service indicators for several cloud computing providers, the proposed method assists to generate a single value of confidence for partners (e.g., a cloud computing provider). The developed indicator will consist of QoS indicators, represented by Key Performance Indicators (KPIs), available in Garg et al. [8] and Sun et al. [9]: availability, response time, stability, security and cost. Therefore the confidence value represents a level of trust in the service provided by cloud computing provider. Hence, a high level of confidence represents a greater probability of the service to be executed without failure during the several stages of VO's life cycle in the cloud. Thus, the proposed model, aims to support the decision process in several stages of VO integration with $\mathrm{CC}$ life cycle, such as the process of cloud computing providers' search and selection or during the operation phase. The generated indicator also can be used and integrated in a reputation service covering all the stages of the VO life cycle.

The remainder of this paper is organized as follows. Section 2 elaborates on the related work and concepts regarding the problem of trust concerning the various processes of a VO. Section 3 seeks to present the proposed model to generate the confidence indicator. Section 4 presents the results of implementation of the model. Finally, Section 5 presents conclusions and future work.

\section{General Background}

This Section presents related work, a definition of cloud computing concept and its relationship to virtual organization. It discusses the concepts of trust and reputation and methods of multi-criteria decision making, and it points out this work contribution regarding related works. 


\subsection{Related Work}

Several works present approaches to support the decision making process to the VO Manager. In this regard, the process of search and selection of service providers based on competencies and candidates' skills occurs through different methods such as: multi-criteria decision making methods and others as optimization by swarm particles [10-12]. These works present methods to enhance the partner's search and selection step during the VO creation, but they do not use or generate a confidence indicator based on Key Performance Indicators (KPIs) belonging to the analyzed partners.

A study presents methods for classification and selection of reliable services based on QoS indicators and user ratings [13] returning a trust indicator. In other studies, services are evaluated according to the observations of users and also on a focused approach through the QoS indicators [14] as well as in satisfaction user ratings used for classification and selection of services $[15,16]$.

The work proposed by Squicciarini et al. [6] presents protocols for trust negotiation representing an effective way to select the best possible members during the several stages of the VO's life cycle. In Winkler et al. [5] it is proposed the generation of a confidence indicator for a reputation service, based on financial, organizational and operational indicators, among others. The developed confidence indicator has served as support for the decision-making tool for partner selection for the composition of the VO.

These aforementioned works seek to meet the purpose of supporting the VO manager in the decision making process in the VO creation phase, specifically in the partners' search and selection step. However, none of them provides an approach which defines a confidence indicator in the context of the virtual organization and cloud computing integration and also do not use this generated confidence indicator to support the several decision-making process presents in VO's life cycle, such as cloud computing partner search and selection and the VO's operation.

The application of confidence indicator on VO, during the integration with cloud computing providers, plays an important role, because the quality of service delivered by cloud providers is a key factor to ensure success in VO's operation. Therefore, the proposed model presents a confidence indicator based on QoS indicators for cloud computing providers, with the purpose to assist the several decision-making process presents in VO's life cycle.

\subsection{Cloud Computing}

The Cloud Computing (CC) paradigm provide access to computing resources (e.g., servers, networks, storage and applications) in a practical way and on-demand, where resources can be provisioned automatically to the user. For this purpose, the Cloud Computing Providers make available the necessary infrastructure so that users can run their services [17]. Also according to the National of Institute and Standards Technology (NIST) [17], CC has four deployment models (public, private, community, hybrid) and can provide three service models: Software as a Service (SaaS), Infrastructure as a Service (IaaS) and Platform as a Service (PaaS). Finally, 
$\mathrm{CC}$ has five essential characteristics (Resource pooling, rapid elasticity, measured service, on-demand self service and broad network access).

Due to the benefits offered by cloud computing, many entities have used their services to implement their applications, so being able to respond quickly and nimbly to a collaboration opportunity [2].

Despite the advantages that cloud computing offers, there are several concerns which fundamentally affect the service quality that providers offer to consumers. Some issues such as trust and security are defined as obstacles to the implementation of services delivered by the cloud computing provider [18]. Although the information related to QoS indicators present in the Service Level Agreement (SLA) is crucial to establishing contracts it is not enough to establish a trust relationship between the provider and the consumer [13], which in the context of $\mathrm{VO}$ and CC integration, the QoS indicators should be monitored, to ensure trust in VO's operation phase. Thus, in order to enhance the quality of the supporting mechanisms for trusting between the entities involved in the VO creation in a CC environment, it is necessary to develop a confidence indicator that can be automatically used by the business partners to make available their services through the resources provided by the cloud computing paradigm.

Particularly, during the several processes of a VO, this confidence indicator can be monitored and updated periodically allowing to identify whether a cloud computing provider continues meeting the needs of trusting regarding the established requirements by the collaboration opportunity. A reputation system using this confidence indicator can support and feed the whole trusting system [19].

\subsection{Trust and Reputation}

The trust concept originates from the social sciences, responsible for the study of the dynamic behavior of human society. Trust is a multidisciplinary concept, studied and applied in psychology, sociology, economics and technology. Psychologists study the trust as a mental attitude and brain behavior when the person trust or distrust someone. Sociologists claim that trust is the social relationship between people [20].

In the information technology area, the trust management problem was introduced by Blaze [21] defined it is and as a collective study of security policies, credentials and trust relationships. The Blaze's approach has resulted in a system, called PolicyMaker that manages and controls access to distributed environments. This system have leveraged several approaches to trust management applied to technological concepts such as e-business environments (Business to Business (B2B), Consumer to Consumer (C2C), Business to Consumer (B2C)), P2P (Peer-to-Peer) and collaborative networks (virtual enterprises) [5, 22, 23].

In addition to multidisciplinary, trust is also a complex concept that has no universally accepted definition. Each scientific area seems to have its own definition. In general, trust can be usually related with levels of confidence in something or someone. Therefore, when dealing with cloud computing paradigm, we can define trust as the level of confidence when using the services provided by the cloud service provider, from the consumer perspective [24]. It is worth noting that, in this work the cloud consumer perspective is associate with the companies that form a VO using 
cloud providers to host their services. Also, it is important to mention that other factors affect the trust of a cloud computing provider, quite often related to mechanisms for protect the user data and unauthorized access. Such factors can be summarized in: security, privacy, accountability and auditability [25]. With the purpose of assisting the trust in a cloud computing environment and other online services the reputation concept can be applied.

The reputation is closely related to the trust. Reputation can be defined as a set of feedback (comments) about the past behavior of the participants of a community, helping in the selection of virtual partners that are considered honest in the real world [26]. The reputation in VO context can be defined as a perception that each business partner has on each member of the VO. The perception is enhanced and developed based on past interactions and objective indicators used to evaluate the collaborative process. The reputation can be seen as a numerical value a participant $\mathrm{A}$ refers to a participant B. Thus, when considering that there is no past transactions, reputation may initially be defined as the value of the confidence indicator in the cloud computing provider.

\subsection{Multiple Criteria Decision Analysis (MCDA)}

Methods of multi-criteria decision-making have emerged as an important ally for solving problems presented by scientific, logistics, engineering and industrial areas. The MCDA can be defined as a collection of methods for matching, classifying and selecting multiple alternatives having multiple attributes whose application depends on the construction of a matrix called assessment matrix or payoff and can also be called scoreboard [27].

The Multiple Criteria Decision Analysis methods can be subdivided into two categories: Multiattribute Utility Theory (MAUT) and Outranking Methods. The MAUT looks for a function that reflects the usefulness of a particular alternative, where each action is assimilated with a level of utility, where the real number represents a preference level of such action. The outranking methods decide which alternative is most appropriate through a pairwise comparison [28].

Among the various MCDA methods presented in Velasquez and Hester [29], the Data Envelopment Analysis method (DEA) will be used because it is capable of handling multiple indicators as cloud providers inputs and outputs and the efficiency of this providers can be quantified and analyzed. DEA is a linear mathematical programming technique for production monitoring, which takes a group of companies and its production indicators (input and output) to assess the productive efficiency of each company decision unit (DMU). Furthermore, it focuses on measuring the efficiency of multiple DMUs in a multiple input and output units environment [30] The Banker, Charnes and Cooper Data Envelopment Analysis (BCC-DEA) model was designed to measure the efficiency of units with variable returns to scale, i.e., increasing the input values of a unit does not affect proportionally the output values [31]. For this reason, the Banker, Charnes and Cooper (BCC) model is suitable for the problem in question and also to the proposed confidence indicator generation model presented in Section 3. Unlike other usual methods to assess confidence, our approach 
uses DEA method because we claim the existence of a connection between efficiency and confidence.

\section{The Proposed Model}

Currently, cloud providers with similar characteristics and different prices have been emerging, eventually due to the competitiveness in the market [8]. In addition, given the diversity of services offered by cloud computing providers, there is a challenge for consumers and, particularly, to the VO Manager, to select a reliable cloud computing provider thereby not causing problems during VO operation. Thus, the proposed confidence indicator can assist the decision making process regarding the search and selection as well as other steps of the VO life cycle.

For this purpose, in the proposed model, Data Envelopment Analysis (DEA) is used to compose a confidence indicator that can be used as a criterion to support VO's manager decisions. Performance indicators that represent Quality of Service will be used for composing the confidence indicator. These indicators belonging to the cloud computing providers will be applied to DEA, which will generate an efficiency level. This efficiency level is reported as the confidence level of that cloud provider. Therefore, the proposed method associates confidence with the Quality of Service delivered by the cloud computing providers.

The rationale relating these concepts is summarized as the higher the efficiency of a cloud provider, the lower the possibility of offering a service that cannot be trusted, given that quality of service indicators will be used, the confidence is related to the quality of service provided by cloud provider. Thus, a high confidence level represents a greater probability of service does not fail, during the VO's operation stage. If the service provided by a cloud computing provider does not attend the need required by the VO's manager, this provider will be replaced preventing issues during the collaboration opportunity attendance.

Fig. 1 depicts the proposed model. The proposed model consists of the following components:

- Cloud Service Providers (CSP): Cloud computing providers that are input to the proposed model based on Quality of Service (QoS) indicators;

- Service Directory: Component responsible for the list of potential candidates as well as the association with the indicators to be used for classification;

- KPIs: Quality of Service indicators of the candidates;

- DEA: linear programming technique which calculates the level of efficiency through candidate KPIs;

- Confidence Indicator: The result of the application of the inputs in the DEA method is a confidence indicator of the cloud computing provider. This indicator can be integrated to the subprocesses present in the VO's creation phase, such as the search and selection step [6]. In that subprocess, the VO manager needs to select a reliable cloud computing provider for the business partner to make available its service in the cloud environment and thus compose the VO. We also foreseen that in addition to providing support in 
the VO creation phase, the confidence indicator can be applied in the VO operation phase, where through a reputation system, it is possible to check if the confidence level of a cloud provider is maintained at an acceptable limit, allowing to perform the providers' change (VO evolution phase) in case the confidence level drops below that limit.

In order to explain the proposed model, Section 3.1 presents the Quality of Service indicators used as input and finally, Section 3.2 details DEA method.

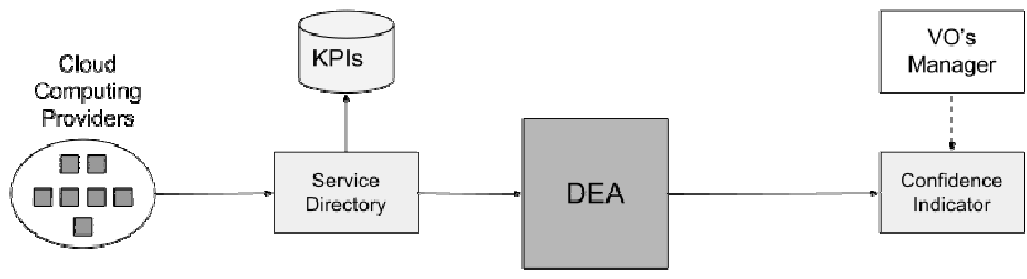

Fig. 1. The proposed model for the confidence indicator generation

\subsection{Key Performance Indicators (KPIs)}

Cloud computing providers can be evaluated using qualitative and quantitative indicators. Qualitative indicators are directly related to the user experience about a service provided. Quantitative indicators can be measured through hardware and software monitoring tools [8].

In order to calculate the confidence level to identify the most suitable service to the user, according to Tang et al. [13] the QoS properties are exploited, which are a set of non-functional attributes of services such as response time, stability, availability, security, etc [8], and these attributes are applicable to all cloud computing service models.

The indicators that will be used to generate a confidence level of a cloud provider are presented in $[8,9]$ :

- Response Time: In the case of services provided in IaaS model, the response time refers to the time that a service is available for use. For instance, the user requests a virtual machine (VM) to the provider, the time is calculated from the moment of the VM creation until it is available for use.

- Stability: The stability of a service is defined as the variance in service performance. According to Garg et al.[8], for cloud storage service, stability is the variance in average time of read and write operations. For computational resources, it is the deviation of performance level specified in SLA, i.e., $\sum_{i=1}^{n} \frac{\frac{\rho_{\text {avg. } i}-\rho_{\text {sla. } i}}{T}}{n}$, where $\rho$ is defined as resource unit (network, storage or computational resource); $\rho_{\text {avg.i }}$ is the monitored average 


\section{L. F. Bilecki and A. Fiorese}

performance of the cloud service leased by user $\mathrm{i} ; \rho_{\text {sla.i }}$ is the promised values in SLA; $\mathrm{T}$ is the service time and $\mathrm{n}$ is the total number of users;

- Security: The security level of a service is a major concerning factor for the protection of security and privacy. It is a qualitative indicator evaluated by service user, which is defined by a numerical value on a scale of 1 to 10 ;

- Availability: It represents the fraction of the time that the service is available to attend requests. Configured as a percentage of service access, given by $\frac{\text { total service time - total service time unavailable }}{\text { total service time }}$.

- Cost: Depends on two attributes: acquisition and on-going. Acquisition cost is used for SaaS services where users sign a service, paying monthly, weekly, among others forms. On-going cost refers to cost of resource usage (cpu, ram, bandwidth or storage) in a unit of time. However, in IaaS providers, cost can be defined as the amount charged for the time granted to use the resources of a VM.

\subsection{DEA Application}

Considering the number of service providers such as $n$ (candidates with their level of confidence to be calculated) and the performance indicators used to evaluate the services, this section present the mathematical model used to calculate the confidence ratio.

Each cloud computing provider is modeled as a Decision Making Unit (DMU). The confidence measure takes into consideration the ratio between the input and output data set, where each input and output is multiplied by its corresponding weight.

Considering that the purpose of this model is the generation of the confidence indicator for cloud computing providers, the input data set corresponds to the Quality of Service indicators (response time, availability, security and stability) and the output data set is defined by the cost indicator. Therefore, we can define confidence as:

$$
z_{0}=\sum_{i=1}^{m} v_{i 0} \times X_{i 0}+v
$$

Subject to,

$$
\begin{aligned}
& \sum_{\mathrm{j}=1}^{\mathrm{s}} \mathrm{u}_{\mathrm{j} 0} \times \mathrm{Y}_{\mathrm{j} 0}=1 \\
& \sum_{i=1}^{m} v_{i k} X_{i k}-\sum_{j=1}^{s} u_{j k} Y_{j k}+v \geq 0 \quad k=\{1,2,3, \ldots, n\}
\end{aligned}
$$


$u_{j}, v_{i} \geq 0 \forall \quad j=1, \ldots, s \quad i=1, \ldots, m$

Where $\mathrm{X}_{\mathrm{ik}}$ and $\mathrm{Y}_{\mathrm{jk}}$ are the input $\mathrm{i}$ and output $\mathrm{j}$ of the DMU k respectively, $\mathrm{v}_{\mathrm{i}}$ and $\mathrm{u}_{\mathrm{j}}$ are the weights; $v$ is a real scale factor; $\mathrm{n}$ are the number of cloud computing providers (DMUs); $\mathrm{m}$ and $\mathrm{s}$, are the total number of input and output indicators, respectively.

Equation 2 sets that the sum of weights multiplied to the value of the output indicator must be equal to 1 due to the BCC-DEA model transformation of fractional to linear, in which confidence value is inversely proportional to output. Inequality 3 defines the confidence value must be positive. Inequality 4 defines the weight of inputs and outputs must be strictly positive. Using the classic BCC-DEA model, the real scale factor in this case is not considered because it determines a return on the production if it is increasing, decreasing or constant to a set of inputs and outputs [32]. Therefore, once calculated the weights, the Equation 5 results the confidence level of the Cloud Computing Provider o :

$$
\text { Confidence }_{o}=\frac{\sum_{j=1}^{s} u_{j 0} \times Y_{j 0}}{\sum_{i=1}^{m} v_{i 0} \times X_{i 0}+v}
$$

\section{Results}

In this Section, the computation of cloud computing providers confidence indicator has been evaluated using QoS indicators of real public service providers: Amazon, RackSpace and Microsoft Azure, available at [8] and used to validate the classification method of cloud computing providers using a MCDA method.

Considering the indicators cited in Section 3.1, Table 1 presents data related to services offered by the following cloud computing providers: Amazon EC2 (CSP1), Rackspace (CSP2) and Azure (CSP3), with their respective values of Quality of Service indicators, weight values related to each indicator for cloud service provider .

Table 1. Offered services, their Quality of Service indicators and relative weight

\begin{tabular}{ccccccc}
\hline KPIs & CSP1 & $\mathbf{W}(\mathbf{1})$ & $\mathbf{C S P 2}$ & $\mathbf{W}(\mathbf{2})$ & $\mathbf{C S P 3}$ & $\mathbf{W}(\mathbf{3})$ \\
\hline Response time & $100 \mathrm{~ms}$ & 0,05 & $600 \mathrm{~ms}$ & 0,05 & $30 \mathrm{~ms}$ & 0,05 \\
Stability & 38,5 & 0,02 & 43 & 0,02 & 49 & 0,02 \\
Security & 4 & 0,05 & 8 & 0,05 & 4 & 0,05 \\
Availability & $99.95 \%$ & 0,7 & $99,99 \%$ & 0,7 & $100 \%$ & 0,7 \\
Cost & $\$ 0.68$ & 1,47 & $\$ 0.96$ & 1,04 & $\$ 0.96$ & 1,04 \\
\hline
\end{tabular}




\section{L. F. Bilecki and A. Fiorese}

The relative weights regarding input and output data sets coping with DEA must be set to define the preferences of the entity responsible for taking decisions regarding the indicators. In other words, weights should be as greater as they are judged as more relevant in the confidence analysis of the DMU. Therefore, only the weight of the cost indicator was set aiming to attend the restriction imposed by the Equality 02 present in the problem formulation and the other weights are randomly assigned to each QoS service attribute.

The application of the indicators in DEA method, returns for each cloud computing provider, the confidence level value related to presented indicators. In Table 2 are presented the results for the method application for each cloud computing provider.

Table 2. Confidence indicator for the presented cloud computing providers

\begin{tabular}{cc}
\hline Cloud Computing Providers & Confidence indicator \\
\hline Amazon EC2 (CSP1) & $14,49 \%$ \\
Rackspace (CSP2) & $3,13 \%$ \\
Azure (CSP3) & $29,54 \%$ \\
\hline
\end{tabular}

According the presented results it is possible to note that a higher value for response time associated with others indicators which represents Quality of Service, cause a decrease in the confidence level.

Considering the VO's and cloud computing integration, where it is necessary to attend a collaboration opportunity, higher response time associated with lower stability of the service provided by the cloud provider, can become issues during the VO's operation. These issues fundamentally affect the collaboration, when it is necessary to respond quickly to a collaboration opportunity in order to follow the market competitiveness.

Moreover, based on the generated confidence indicator, the VO's manager can establish an acceptable confidence limit and then select the most reliable cloud computing provider to host the VO's members services,

\section{Conclusion and Future Work}

This article presents a model to generate a confidence indicator for cloud computing providers based on Key Performance Indicators comprising Quality of Service measures. Also, this model can be adapted to several scenarios that do not use cloud computing providers, just changing the indicators' set. The generation of this confidence indicator is driven by the problem observed during the subprocesses of VO's life cycle, where is necessary the establishment of confidence between involved members, so that the confidence is a crucial factor in VO and Cloud Computing integration.

The presented results show that the proposed method has the potential to generate a confidence indicator that can support cloud computing providers' selection in the VO creation stage. The VO's manager can see which cloud computing providers are most reliable and appropriate to integrate $\mathrm{VO}$, based on generated confidence indicator from Quality of Service values. Furthermore, the proposed method can be customized by adding more indicators to compose the confidence indicator. Also, the VO's 
manager can choose weight values, giving priority to the desired indicators for every VO Collaboration Opportunity.

In a further work, we intend to integrate this confidence indicator model in a reputation architecture, composed of a reputation system that will carry out the monitoring and evaluation of each entity present in the Virtual Organization composed by services host in cloud providers. Also, it is intended to investigate how this reputed confidence indicator can support the Virtual Organization Manager in the several stages of VO's life cycle.

\section{References}

1. Esposito, E., Evangelista, P.: Investigating virtual enterprise models: Literature review and empirical findings. Int. J. Prod. Econ. 148, 145-157 (2014).

2. Camarinha-Matos, L.M., Afsarmanesh, H., Galeano, N., Molina, A.: Collaborative networked organizations - Concepts and practice in manufacturing enterprises. Comput. Ind. Eng. 57, 46-60 (2009).

3. de Lemos, F.S.B., Fiorese, A., Alves O.C Jr., Vieira, R.G.: Using data envelopment analysis and fuzzy logic as intelligent risk-based decision making support for virtual organizations. Intell. Syst. Ref. Libr. 87, 203-218 (2015).

4. Camarinha-Matos, L.M., Afsarmanesh, H.: The Virtual Enterprise Concept. Infrastructures Virtual Enterprise. 153, 3-14 (1999),Springer.

5. Winkler, T.J., Haller, J., Gimpel, H., Weinhardt, C.: Trust Indicator Modeling for a Reputation Service in Virtual Organizations. ECIS 2007 Proc. 1584-1595 (2007).

6. Squicciarini, A.C., Paci, F., Bertino, E.: Trust establishment in the formation of Virtual Organizations. Comput. Stand. Interfaces. 33, 13-23 (2011).

7. Vieira, R.G., Alves-Junior, O.C., Rabelo, R.J., Fiorese, A.: A Risk Analysis Method to Support Virtual Organization Partners' Selection. Presented at the (2014).

8. Garg, S.K., Versteeg, S., Buyya, R.: A framework for ranking of cloud computing services. Futur. Gener. Comput. Syst. 29, 1012-1023 (2013).

9. Sun, M., Zang, T., Xu, X., Wang, R.: Consumer-centered cloud services selection using AHP. In: Service Sciences (ICSS), 2013 International Conference on. pp. 1-6 (2013).

10.Junior, O.C.A., Rabelo, R.J.: A KPI Model for Logistics Partners' Search and Suggestion to Create Virtual Organisations. Int. J. Netw. Virtual Organ. 12, 149-177 (2013).

11.Sadigh, B.L., Arikan, F., Ozbayoglu, a. M., Unver, H.O., Kilic, S.E.: A Multi-agent System Model for Partner Selection Process in Virtual Enterprise. Procedia Comput. Sci. 36, 367 372 (2014).

12.Zhao, Q., Zhang, X., Xiao, R.: Particle swarm optimization algorithm for partner selection in virtual enterprise. Prog. Nat. Sci. 18, 1445-1452 (2008).

13.Tang, M., Dai, X., Liu, J., Chen, J.: Towards a trust evaluation middleware for cloud service selection. Futur. Gener. Comput. Syst. (2016).

14.Noor, T.H., Sheng, Q.Z., Ngu, A.H.H., Alfazi, A., Law, J.: Cloud Armor: a platform for credibility-based trust management of cloud services. Proc. 22nd ACM Int. Conf. Inf. Knowl. Manag. 2509-2512 (2013).

15.Yau, S.S., Yin, Y.: QoS-based service ranking and selection for service-based systems. Proc. - 2011 IEEE Int. Conf. Serv. Comput. SCC 2011. 56-63 (2011).

16.Liu, X., Fletcher, K.K., Tang, M.: Service selection based on personalized preference and trade-offs among QoS factors and price. Proc. - 2012 IEEE 1st Int. Conf. Serv. Econ. SE 2012. 32-39 (2012). 


\section{L. F. Bilecki and A. Fiorese}

17.Mell, P.M., Grance, T.: SP 800-145. The NIST Definition of Cloud Computing. National Institute of Standards \& Technology, Gaithersburg, MD, United States (2011).

18.Hwang, K., Li, D.: Trusted cloud computing with secure resources and data coloring. IEEE Internet Comput. 14, 14-22 (2010).

19.Mashayekhy, L., Grosu, D.: A reputation-based mechanism for dynamic virtual organization formation in grids. Proc. Int. Conf. Parallel Process. 108-117 (2012).

20.Cho, J.-H., Swami, A., Chen, I.-R.: A survey on trust management for mobile ad hoc networks. IEEE Commun. Surv. Tutorials Tutorials. 13, 562-583 (2011).

21.Blaze, M., Feigenbaum, J., Lacy, J.: Decentralized trust management. Secur. Privacy, 1996. Proceedings., 1996 IEEE Symp. 164-173 (1996).

22.Kerschbaum, F., Haller, J., Karabulut, Y., Robinson, P.: PathTrust: A trust-based reputation service for virtual organization formation. Lect. Notes Comput. Sci. (including Subser. Lect. Notes Artif. Intell. Lect. Notes Bioinformatics). 3986 LNCS, 193-205 (2006).

23.Resnick, P., Zeckhauser, R.: Trust among strangers in Internet transactions: Empirical analysis of eBay's reputation system. Adv. Appl. ... 127-157 (2002).

24.Ko, R.K.L., Jagadpramana, P., Mowbray, M., Pearson, S., Kirchberg, M., Liang, Q., Lee, B.S.: TrustCloud: A framework for accountability and trust in cloud computing. Proc. 2011 IEEE World Congr. Serv. Serv. 2011. 584-588 (2011).

25.Pearson, S., Benameur, A.: Privacy, Security and Trust Issues Arising from Cloud Computing. 2010 IEEE Second Int. Conf. Cloud Comput. Technol. Sci. 693-702 (2010).

26.Comput, J.P.D., Hendrikx, F., Bubendorfer, K., Chard, R.: Reputation systems : A survey and taxonomy. J. Parallel Distrib. Comput. 75, 184-197 (2015).

27.Rehman, Z.U., Hussain, O.K., Hussain, F.K.: Iaas cloud selection using MCDM methods. Proc. - 9th IEEE Int. Conf. E-bus. Eng. ICEBE 2012. 246-251 (2012).

28.Whaiduzzaman, M., Gani, A., Anuar, N.B., Shiraz, M., Haque, M.N., Haque, I.T.: Cloud service selection using multicriteria decision analysis. Sci. World J. 2014, 10 (2014).

29.Velasquez, M., Hester, P.: An Analysis of Multi-Criteria Decision Making Methods. Int. J. Oper. Res. .... 10, 56-66 (2013).

30.Chen, W.C., Johnson, A.L.: A unified model for detecting efficient and inefficient outliers in data envelopment analysis. Comput. Oper. Res. 37, 417-425 (2010).

31.Banker, R.D., Charnes, A., Cooper, W.W.: Some models for estimating technical and scale inefficiencies in data envelopment analysis. Manage. Sci. 30, 1078-1092 (1984).

32.Souza, L.M. De: Performance Evaluation Methodology for Cloud Computing using Data Envelopment Analysis. 58-64 (2015). 